\title{
Schedule-induced polydipsia contrast in the rat
}

\author{
JOSEPH H. PORTER \\ Virginia Commonwealth University, Richmond, Virginia 23284 \\ and \\ JOSEPH D. ALLEN \\ University of Georgia, Athens, Georgia 30602
}

\begin{abstract}
Schedule-induced polydipsia was studied using a behavioral contrast paradigm. Food pellets were delivered to food-deprived rats on a response-independent FT 1-min schedule. Licking on a tube produced water on a MULT FR $10 \mathrm{FR} 10$, MULT FR 10 EXT, or MIXED FR 10 EXT for three rats (Experiment 1) and on a MULT VI VI, MULT VI EXT, or MIXED VI EXT schedule for three other rats (Experiment 2). On the FR schedules, rats could drink more water by increasing lick rates, but on the VI schedules the amount of drinking was fixed by the schedule parameters and was relatively unaffected by lick rates. Relative to MULT FR FR, positive polydipsia contrast was clearly demonstrated on MULT and MIXED FR EXT; but relative to MULT VI VI, contrast was not demonstrated on MULT and MIXED VI EXT. These data suggest that polydipsia contrast occurs only if increased licking permits increased drinking.
\end{abstract}

Several studies (Allen, Porter, \& Arazie, 1975; Jacquet, 1972; Porter, Arazie, Holbrook, Cheek, \& Allen, 1975) have reported contrast-like effects with schedule-induced polydipsia (Falk, 1969, 1971). However, none employed the standard multiple schedule paradigm used to investigate behavioral contrast (see Reynolds, 1961). Recently, Allen and Porter (1975) demonstrated positive polydipsia contrast using a procedure analogous to the standard behavioral contrast design. Food pellets were delivered on a fixed-interval (FI) 1-min schedule. Drinking was initially stabilized on a MULT CRF CRF schedule (schedule components lasted $1 \mathrm{~min}$ ), and when access to water was prevented in one component (MULT CRF EXT), drinking increased substantially in the unchanged component. When access to water was reinstated in the EXT component, drinking in the unchanged component decreased to previous levels.

The Allen and Porter (1975) study is subject to two criticisms. First, a leverpress response was required to deliver $.1 \mathrm{ml}$ of water in a water dipper, rather than the more direct response of licking a drinking tube. This method apparently did not constrain the development of schedule-induced polydipsia, since

This study was supported by Contract DADA17-73-3007 from the U.S. Army Research and Development Command to B. N. Bunnell, I. S. Bernstein, and J. D. Allen. The authors wish to thank Wayne R. Lowther and Steven F. Peed for their assistance in the conduct of this study. Portions of this study were presented at the 1975 meeting of the Psychonomic Society in Denver, Colorado. Requests for reprints should be sent to Joseph $\mathrm{H}$. Porter, Department of Psychology, Virginia Commonwealth University, Richmond, Virginia 23284. milliliter/pellet intake values were within previously reported ranges. Second, the water-reinforcement schedule (CRF) permitted the amount of water received by the rats to increase during the unchanged component. It might be argued that the increased water intake, rather than the contrast condition, accounted for the increased leverpress rates in the unchanged component. This was unlikely, since leverpress rates (and drinking magnitudes) returned to baseline levels when the MULT CRF CRF schedule was reinstated.

The present study was designed to avoid these criticisms. In Experiment 1, licking was substituted for leverpressing as the response that produced water, and licking was reinforced by water delivery on a fixed-ratio (FR) 10 schedule. This low schedule value allowed the rats relatively unlimited access to water, and increases in water consumption could occur in the unchanged component of the MULT schedule, as they had in the CRF component of the Allen and Porter (1975) study. In Experiment 2, licking was reinforced on variable interval (VI) schedules, which prevented any increase in water consumption during the unchanged component of the MULT schedule.

\section{EXPERIMENT 1: DRINKING CONTRAST}

\footnotetext{
Method

Subjects. Three adult female hooded rats served as subjects. Rat 0P3 had previous experience with fixed-interval (FI) food reinforcement schedules and with schedule-induced polydipsia; rats 661 and 662 were naive. They were housed individually, had free access to water in the home cages throughout the study,
} 
and were maintained at $75 \%-80 \%$ of their free feeding weights by adjusting their daily ration of Purina Rat Chow.

Apparatus. A Lehigh Valley Electronics operant conditioning chamber (Model 1417) with a sound-attenuated cubicle was used. The food magazine was located where the right-hand lever would normally be on the intelligence panel, and no response levers were present. Water was delivered by a BRS/LVE liquid solenoid valve (Model 114-06) located behind the intelligence panel. Access to the water was through a 35 -mm-diam aperture in the front panel $40 \mathrm{~mm}$ to the left of the food magazine and $20 \mathrm{~mm}$ above the floor of the chamber. The water trough was recessed about $10 \mathrm{~mm}$ behind the aperture. Licks were recorded with a GrasonStadler drinkometer (Model E490 A). A Ralph Gerbrands (Model D) pellet dispenser delivered 45-mg standard formula Noyes pellets. There was a $7-\mathrm{W}$ houselight $(20 \mathrm{~V} / \mathrm{ac})$ and a $2.5-\mathrm{kHz}$ tone which raised the ambient white noise level in the chamber from $52 \mathrm{~dB}$ to $70 \mathrm{~dB}$. Standard electromechanical programming and recording equipment was located in an adjacent room.

Procedure. Food pellets were delivered independently of responding on a fixed-time 1-min (FT 1-min) schedule throughout the study. Each session consisted of 601 -min components. Sessions were conducted 5 days per week, and the rats were maintained at their adjusted weights over weekends. The solenoid valve was calibrated to deliver $.05 \mathrm{ml}$ of water with each operation. Initially, water delivery was placed on a FR 6 reinforcement schedule in which every sixth lick produced $.05 \mathrm{ml}$ of water. The schedule was increased to FR 10 after one or two sessions, and water delivery was placed on a multiple (MULT) FR 10 FR 10 reinforcement schedule (1-min components). Half of the 601 -min components were randomly paired with the light and tone on $(\mathrm{L}+\mathrm{T})$, the other half with light and tone off $(\overline{\mathrm{L}}+\overline{\mathrm{T}})$. After drinking in both components was judged to be relatively stable, the water-reinforcement schedule was changed to MULT FR 10 extinction (EXT). During the EXT components, licks on the water trough were recorded, but did not operate the solenoid valve. For rats $O P 3$ and $662, L+T$ signaled extinction, and $\bar{L}+\bar{T}$ signaled water reinforcement. For rat 661 , the cue contingencies were reversed. Next, the water-reinforcement schedule was changed to MIXED FR 10 EXT in which either $L+T$ was present throughout all components (rats 661 and $0 \mathrm{P} 3$ ) or $\overline{\mathrm{L}}+\overline{\mathrm{T}}$ was present throughout all components (rat 662). Licks were still recorded separately for the reinforced components and the extinction components. The schedule was returned to MULT FR 10 EXT and then a MULT FR 10 FR 10 in order to recapture baseline performance. Finally, the water-reinforcement schedule was made MULT EXT EXT (the 1-min food delivery schedule continued). Table 1 shows the number of sessions per rat per condition and the order of conditions.

\section{Results and Discussion}

Figure 1 shows the mean licks per 1-min interval for both $\mathrm{L}+\mathrm{T}$ and $\overline{\mathrm{L}}+\overline{\mathrm{T}}$ components of each multiple schedule. Data during the mixed schedules are presented as if there were $L+T$ and $\bar{L}+\bar{T}$ components, although only one cue condition was present throughout each session. Only the last eight sessions of the initial MULT FR 10 FR are shown, and for all three rats, lick rates were similar across the two components. On MULT FR 10 EXT, licking decreased to low levels in the changed component (EXT), and increased during the unchanged component (FR 10), thus demonstrating positive polydipsia contrast.

On MIXED FR 10 EXT, polydipsia contrast was still evident, showing that the presence of discriminative cues was not necessary to maintain contrast (Gonzalez \& Champlin, 1974; Scull, Davies, \& Amsel, 1970). Also, there were no systematic changes in lick rates when the discriminative cues were removed.

Lick rates for all three rats returned to baseline levels when the MULT FR 10 FR 10 schedule was reinstated. In the final schedule condition, both components were associated with extinction for 15 sessions, and all three rats maintained low, but consistent, lick rates for the entire 15 sessions. In fact, rats $0 P 3$ and 661 maintained lick rates during MULT EXT EXT which were as high or higher than those produced during the EXT components of the MULT FR 10 EXT schedule. These findings suggest that licking in EXT components of the MULT FR 10 EXT schedule was not due to induction from water-reinforced drinking in the FR 10 condition, but was perhaps scheduled-induced "licking"' maintained by the FT 1-min reinforcement schedule.

Mean milliliter/interval/component water intakes for the last five sessions of each condition are shown in Table 1 . They were computed by multiplying $0.05 \mathrm{ml}$ times the number of water reinforcers. Water spillage was aportioned between the two FR components and

Table 1

Number of Sessions on Each Reinforcement Schedule for Each Subject in Experiment 1

\begin{tabular}{|c|c|c|c|c|c|c|c|c|c|}
\hline \multirow[b]{2}{*}{$\begin{array}{c}\text { Reinforcement } \\
\text { Schedule }\end{array}$} & \multicolumn{3}{|c|}{ OP3 } & \multicolumn{3}{|c|}{661} & \multicolumn{3}{|c|}{662} \\
\hline & $\begin{array}{c}\text { Number of } \\
\text { Sessions }\end{array}$ & $\mathbf{L}+\mathrm{T}$ & $\overline{\mathrm{L}}+\overline{\mathrm{T}}$ & $\begin{array}{c}\text { Number of } \\
\text { Sessions }\end{array}$ & $L+T$ & $\overline{\mathbf{L}}+\overline{\mathrm{T}}$ & $\begin{array}{c}\text { Number of } \\
\text { Sessions }\end{array}$ & $L+T$ & $\overline{\mathrm{L}}+\overline{\mathrm{T}}$ \\
\hline MULT FR 10 FR 10 & 33 & .40 & .37 & 25 & .16 & .16 & 25 & .43 & .40 \\
\hline MULT FR 10 EXT & 10 & & .65 & 20 & .57 & & 31 & & 1.03 \\
\hline MIXED FR 10 EXT & 10 & .60 & & 10 & .72 & & 10 & & 1.13 \\
\hline MULT FR 10 EXT & 10 & & .57 & 15 & .77 & & 10 & & 1.03 \\
\hline MULT FR10 FR 10 & 6 & .41 & .42 & 18 & .25 & .24 & 12 & .55 & .50 \\
\hline MULT EXT EXT & 15 & & & 14 & & & 14 & & \\
\hline
\end{tabular}

Note-Mean $\mathrm{ml} /$ interval water intake for the last five sessions of each schedule is shown for light-and-tone-on ( $L+T)$ and light-andtone-off $(\bar{L}+\bar{T})$ intervals. Water intake on MIXED FR IOEXT is shown under the cue condition which was present throughout the session. 


\section{DRINKING CONTRAST}

R-OP3

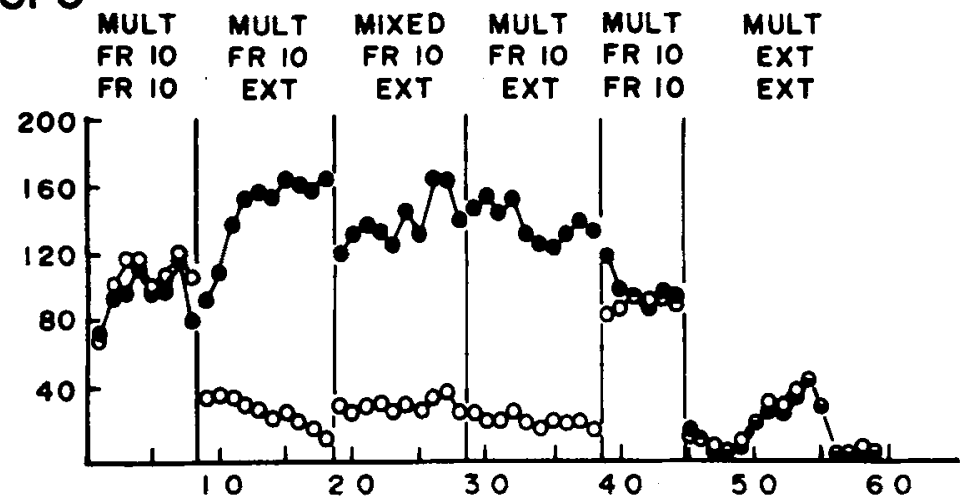

R-66I

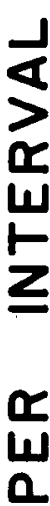

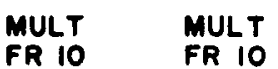

$\begin{array}{cccccc}\text { FR } 10 & \text { FR 10 } & \text { FR IO } & \text { FR IO } & \text { FR 10 } & \text { EXT } \\ \text { FR 10 } & \text { EXT } & \text { EXT } & \text { EXT } & \text { FR 10 } & \text { EXT }\end{array}$

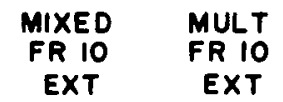

MULT
R 10
R 10

MULT

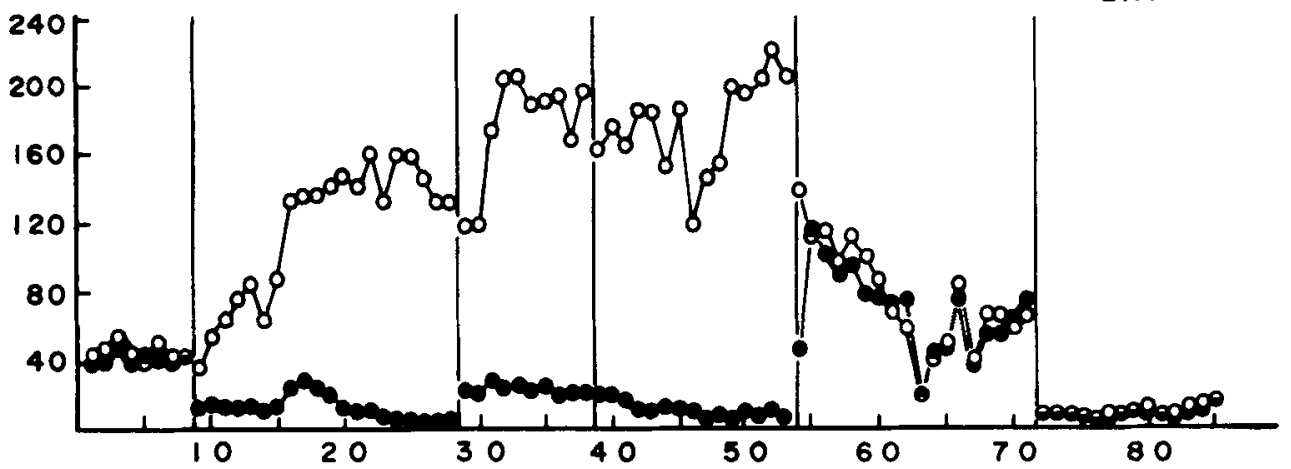

R-662

$\stackrel{0}{y}$

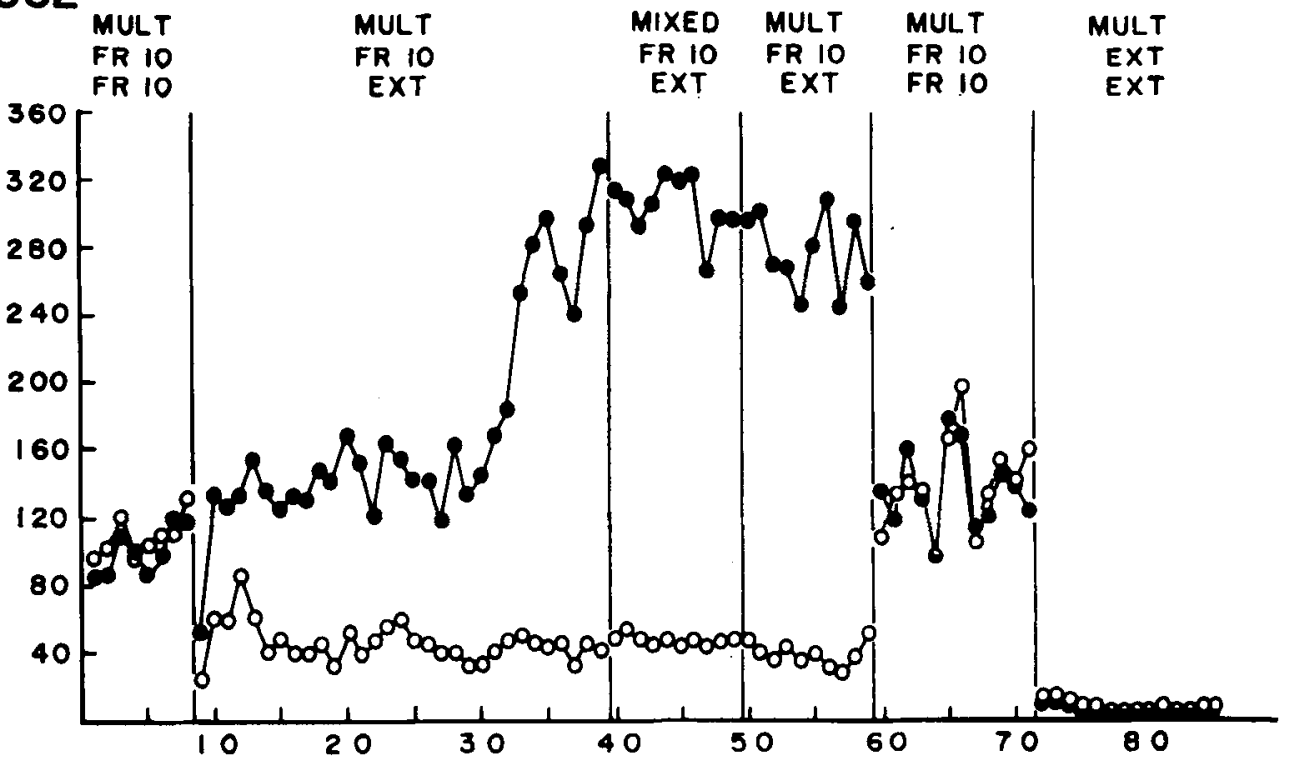

\section{SESSIONS}

Figure 1. Mean licks per interval during light-plus-tone-on (open circles) and light-plus-tone-off (closed circles) components for each schedule condition. 


\section{DRINKING CONTRAST}

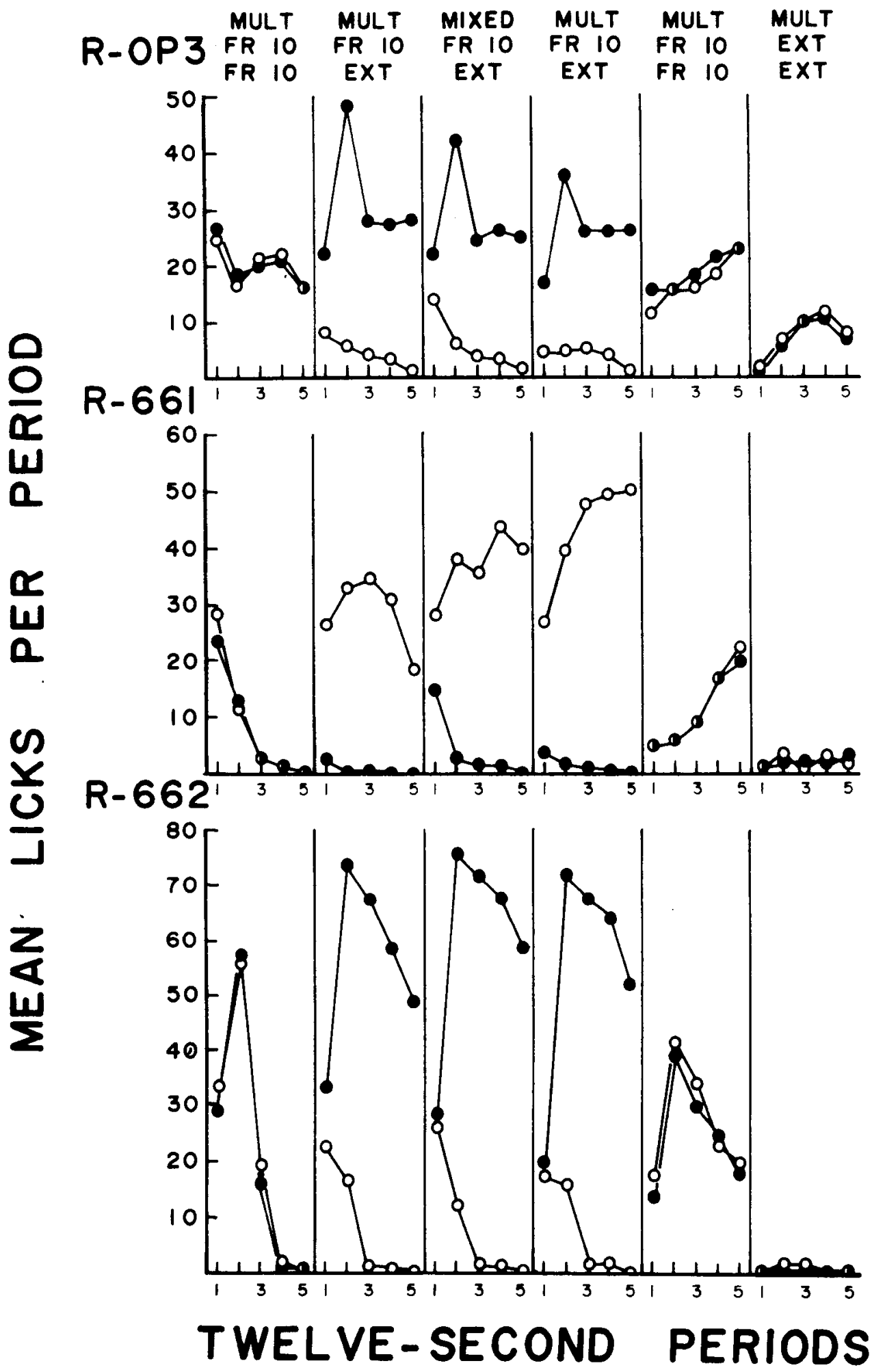

Figure 2. Lick distributions during light-plus-tone-on (open circles) and light-plus-tone-off (closed circles) components of the last five sessions of each condition. 
Table 2

Number of Sessions on Each Reinforcement Schedule for Each Subject in Experiment 2

\begin{tabular}{|c|c|c|c|c|c|c|c|c|c|c|c|}
\hline \multicolumn{4}{|c|}{663} & \multicolumn{4}{|l|}{664} & \multicolumn{4}{|l|}{665} \\
\hline $\begin{array}{l}\text { Reinforcement } \\
\text { Schedule }\end{array}$ & $\begin{array}{c}\text { Number } \\
\text { of Ses- } \\
\text { sions }\end{array}$ & $L+T$ & $\overline{\mathrm{L}}+\overline{\mathrm{T}}$ & $\begin{array}{l}\text { Reinforcement } \\
\text { Schedule }\end{array}$ & $\begin{array}{c}\text { Number } \\
\text { of Ses- } \\
\text { sions }\end{array}$ & $\mathrm{L}+\mathrm{T}$ & $\overline{\mathrm{L}}+\overline{\mathrm{T}}$ & $\begin{array}{l}\text { Reinforcement } \\
\text { Schedule }\end{array}$ & $\begin{array}{c}\text { Number } \\
\text { of Ses- } \\
\text { sions }\end{array}$ & $\mathrm{L}+\mathrm{T}$ & $\overline{\mathrm{L}}+\overline{\mathrm{T}}$ \\
\hline MULT VI1 2 VI 12 & 43 & .12 & .12 & MULT VI 22.5 VI 22.5 & 31 & .12 & .13 & MULT VI 22.5 VI 22.5 & 38 & .12 & .11 \\
\hline MULT VI1 2 EXT & 15 & & .16 & MULT VI 22.5 EXT & 26 & .10 & & MULT VI $22.5 \mathrm{EXT}$ & 30 & .11 & \\
\hline MIXED VI 12 EXT & 13 & & .16 & MULT VI 22.5 EXT & 10 & & .07 & MIXED VI 22.5 EXT & 7 & .11 & \\
\hline MULT VI 12 EXT & 6 & & .16 & MULT VI 22.5 EXT & 8 & .10 & & MULT VI 22.5 VI 22.5 & 15 & .08 & .09 \\
\hline MULT VI 12 VI 12 & 26 & .18 & .17 & MULT VI 22.5 VI 22.5 & 12 & .07 & .05 & MULT VI 22.5 EXT & 5 & & .11 \\
\hline MULT VI 12 EXT & 15 & & .20 & MULT VI 22.5 EXT & 5 & & .05 & MULT VI 12 VI 12 & 13 & .17 & .18 \\
\hline MULT VI 12 VI 12 & 15 & .18 & .17 & MUIT VI 12 VI 12 & 16 & .14 & .13 & MULT VI $12 \mathrm{EXT}$ & 15 & .15 & \\
\hline \multirow[t]{3}{*}{ MULT EXT EXT } & 10 & & & MULT VI 12 EXT & 15 & & .15 & MULT VI 12 VI 12 & 10 & .18 & .16 \\
\hline & & & & MULT VI 12 VI 12 & 10 & .16 & .15 & MULT EXT EXT & 10 & & \\
\hline & & & & MULT EXT EXT & 10 & & & & & & \\
\hline
\end{tabular}

Note-Mean ml/interval water intake for the last five sessions of each schedule is shown for light-and-tone-on ( $L+T)$ and light-andtone-off $(\bar{L}+\bar{T})$ intervals. Water intake on MIXED VI EXT is shown under the cue condition which was present throughout the session.

then subtracted before water intakes were computed. These data closely parallel the licks per interval data presented in Figure 1. Thus, water consumption increased during the unchanged component in the contrast condition, but returned to baseline level when the MULT FR 10 FR 10 schedule was reinstated.

Figure 2 shows the distribution of licking within 1-min interpellet intervals of $L+T$ and $\bar{L}+\bar{T}$ for each schedule in the experiment. Lick rate was highest in the first or second 12-sec period of the interval during the initial MULT FR 10 FR 10 schedule. The lick distributions demonstrate that the increased water consumption during the FR components of the multiple and mixed schedules resulted from an elevation in and extension of drinking in the last four 12 -sec intervals. Upon return to MULT FR 10 FR 10, the distributions of licking approximated, but did not recapture, the distributions produced by the first MULT FR 10 FR 10 condition. During the MULT EXT EXT schedule, lick rates for rats 661 and 662 were low and were relatively constant over all five 12 -sec periods of the interval. The lick distribution for rat $0 \mathrm{P} 3$ peaked during the fourth period of the interval.

\section{EXPERIMENT 2: LICKING CONTRAST}

\footnotetext{
Method

Subjects and Apparatus. Three adult naive female hooded rats served as subjects. Housing, maintenance conditions, and apparatus were the same as in Experiment 1.

Procedure. As in Experiment 1, food pellets were delivered on an FT 1-min schedule throughout the study, and each session consisted of $601-\mathrm{min}$ components. Sessions were conducted daily, 5 days per week. Initially, every sixth lick (FR 6) produced $.05 \mathrm{ml}$ of water. The schedule was increased to FR 10 after the first or second sessions. After 10 sessions on FR schedules, water delivery was placed on a MULT variable-interval (VI) 12-sec variable-interval 12 -sec reinforcement schedule (1-min compo-
}

nents). Half of the 601 -min components were randomly paired with $\mathbf{L}+T$, the other half with $\overline{\mathbf{L}}+\overline{\mathbf{T}}$. After eight sessions, the water schedule was changed to MULT VI $22.5 \mathrm{sec}$ VI $22.5 \mathrm{sec}$. Rat 663 did not maintain schedule-induced drinking on this schedule and was returned to MULT VI $12 \mathrm{sec}$ VI $12 \mathrm{sec}$ after five sessions. Rats 664 and 665 remained on the MULT VI 22.5.sec VI 22.5-sec schedule. After drinking in both components was judged to be relatively stable, the water reinforcement schedule was changed to MULT VI EXT. Table 2 shows the number of sessions received by each rat under each condition, and the order of presentation for each condition. Table 2 also shows the cue contingencies for each rat. Rats 664 and 665 were later tested on the MULT VI 12-sec VI 12-sec water reinforcement schedule to assess the effects of the two different VI reinforcement schedules.

\section{Results and Discussion}

Figure 3 shows the mean licks per interval for both $\mathrm{L}+\mathrm{T}$ and $\overline{\mathrm{L}}+\overline{\mathrm{T}}$ components of each multiple schedule. Data during the mixed schedules are presented as if there were $\mathrm{L}+\mathrm{T}$ and $\overline{\mathrm{L}}+\overline{\mathrm{T}}$ components, although only one cue condition was present throughout each session (see Table 2).

When rat 663 was switched from MULT VI 12 -sec VI 12-sec to MULT VI 12-sec EXT, lick rates increased slightly during the VI 12-sec components and decreased during the EXT component. The elevated lick rates were maintained in the unchanged components during subsequent MIXED and MULT VI 12-sec EXT conditions. However, on return to MULT VI $12 \mathrm{sec}$ VI $12 \mathrm{sec}$, licks per interval remained at an elevated level during the unchanged component and increased to this level in the former EXT component. Thus, baseline lick rates were not recaptured. When returned to MULT VI 12-sec EXT, rat 663 once again displayed what appeared to be positive polydipsia contrast, but baseline lick rates were not recaptured when the MULT VI 12-sec VI 12-sec schedule was reinstated. Premack (1969) has also reported an instance where rats failed to return to baseline performance and the elevated level 
LICKING CONTRAST

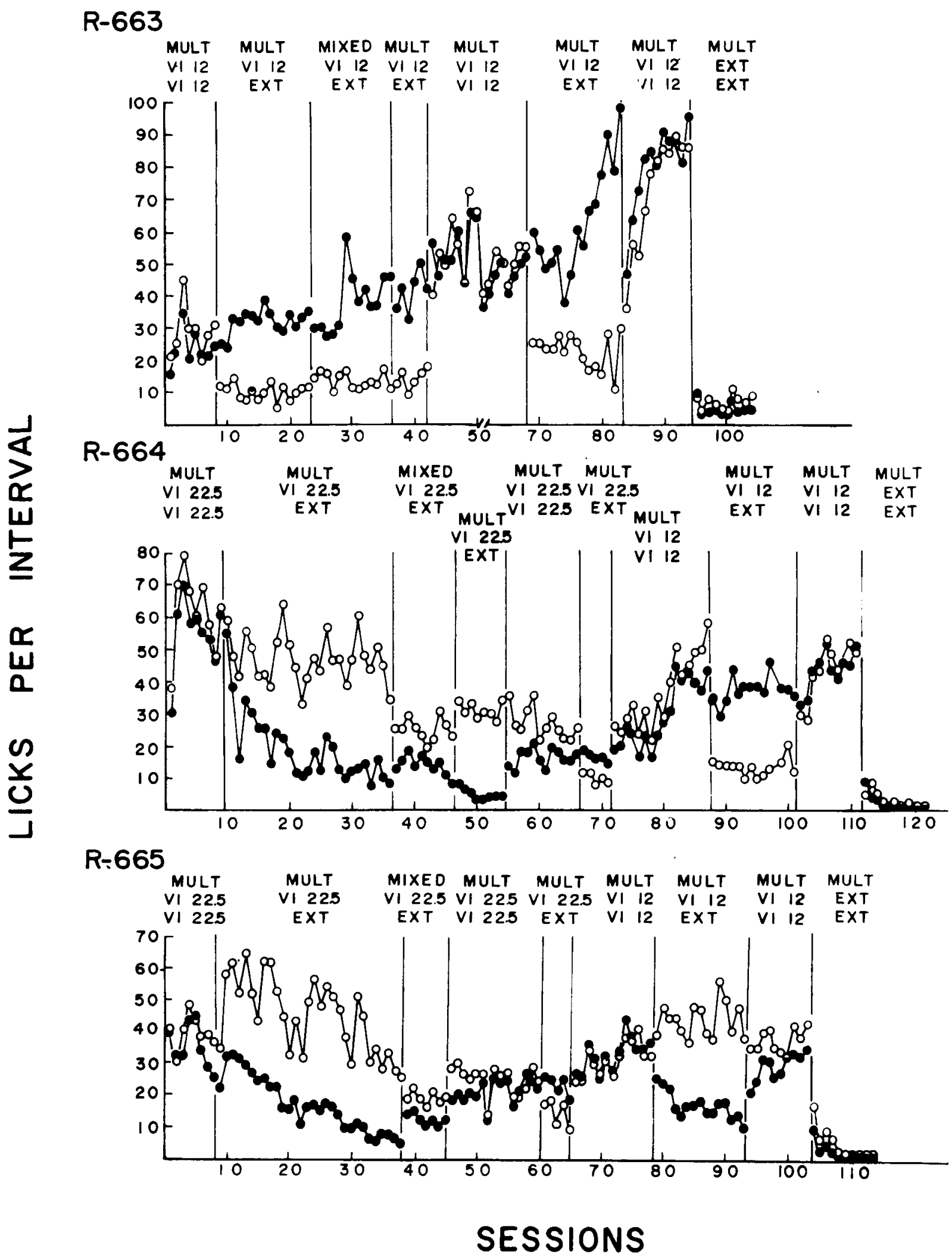

Figure 3. Mean licks per interval during light-plus-tone-on (open circles) and light-plus-tone-off (closed circles) components for each schedule condition. 
of behavior in the unchanged component was maintained.

Rat 664 displayed negative induction when placed on MULT VI 22.5-sec EXT, and the decreased lick rates in both components continued across the subsequent MIXED and MULT VI 22.5-sec EXT conditions. Lick rates in both components on MULT VI $22.5 \mathrm{sec}$ VI $22.5 \mathrm{sec}$ were allowed to stabilize again, and then the schedule was changed to MULT VI 22.5-sec EXT. Very little change was evident during the constant component. Lick rates in both components increased when the schedule was changed to MULT VI $12 \mathrm{sec}$ VI $12 \mathrm{sec}$ and, when the schedule was switched to MULT VI 12-sec EXT, a slight negative induction effect was again shown. Upon return to MULT VI $12 \mathrm{sec}$ VI $12 \mathrm{sec}$, baseline lick rates were recaptured.

When rat 665 was switched from MULT VI $22.5 \mathrm{sec} 22.5 \mathrm{sec}$ to MULT VI 22.5-sec EXT, licking in the unchanged component increased substantially but returned to baseline levels by the end of 30 sessions. Licking decreased even further during the MIXED schedule condition. Transient positive contrast has been reported previously by Pear and Wilkie (1971) with rats and by Terrace (1968) with pigeons. They found that if discrimination training on a MULT VI EXT schedule was long enough, the positive contrast effect gradually decreased until response rate in the unchanged component approximated the baseline rate. Positive contrast was not evident during the second MULT VI 22.5-sec EXT condition. However, stable positive contrast did occur when the MULT VI 12-sec VI 12-sec baseline was changed to MULT 12-sec EXT. Furthermore, baseline lick rates were recaptured when the MULT VI 12-sec VI 12-sec schedule was reinstated.

As in Experiment 1, when the MULT EXT EXT schedule was instated, lick rates fell to very low, but stable about-zero, levels for all three rats. Once again, this behavior appeared to resemble scheduleinduced licking of the dry tube. Mendelson and Chillag (1970) reported that schedule-induced licking occurred when a cold closed stainless steel drinking tube was made available. The stainless steel water tube in the present study apparently assumed the same function.

Mean milliliter/interval water intakes (obtained in the same manner as in Experiment 1) for the last five sessions of each condition are shown in Table 2 . Water spillage was subtracted before milliliter/ interval intake was computed. The data show that there was very little change in water consumption during the unchanged components when the schedule was changed from MULT VI VI to MULT VI EXT. In fact, rat 665 showed a slight decrease in milliliter/ interval intake during the two instances where posi- tive polydipsia contrast was displayed (the first MULT VI 22.5-sec EXT schedule and the MULT VI 12-sec EXT schedule).

Figure 4 shows the distribution of licking within the 1-min interpellet intervals of $L+T$ and $\bar{L}+\bar{T}$ for each schedule in the experiment. Rats 664 and 665 displayed licking patterns that were characteristic of post-pellet drinking in spite of the fact that the probability of water delivery was low and constant within the interpellet interval. The licking distributions for rat 663 were initially flat and in line with the scheduled distribution of water delivery on the VI schedule. With continued exposure, however, licking became more positively accelerated within the interval, assuming a pattern typified by foodreinforced operants during FI schedules. This is very similar to a previously reported instance of scheduleinduced drinking that turned into FI operant-like drinking (Segal, 1969). During the MULT EXT EXT condition, lick distributions during both components for all three rats were relatively flat.

\section{GENERAL DISCUSSION}

While the present results demonstrate that positive behavioral contrast can be obtained with scheduleinduced polydipsia, its occurrence may depend upon the water reinforcement schedule (ratio vs. interval). In Experiment 1 when a MULT FR 10 FR 10 schedule was used, all three rats displayed positive polydipsia contrast when switched to MULT FR 10 EXT. When the MULT FR 10 FR 10 schedule was reinstated, licking and water intake in the constant component returned to original baseline levels. This schedule of reinforcement allowed the rats to have relatively unlimited access to water, thus approximating a normal schedule-induced polydipsia situation where water is available continuously. These results paralleled those of Allen and Porter (1975), who obtained polydipsia contrast when water was continuously available during the constant component of a MULT CRF EXT schedule. When water availability was restricted by reinforcing licking on VI schedules (Experiment 2), the only clearcut demonstration of polydipsia contrast was obtained on a MULT VI 12-sec EXT schedule by rat 665 . Rat 663 showed what appeared to be polydipsia contrast on each MULT VI 12-sec EXT condition, but failed to return to baseline levels when returned to MULT VI $12 \mathrm{sec}$ VI $12 \mathrm{sec}$. When VI 22.5-sec water reinforcement schedules were used, rat 665 displayed a transient polydipsia contrast, while rat 664 displayed negative induction. Thus, there was no reliable demonstration of polydipsia contrast on MIXED or MULT VI EXT schedules.

One possible explanation for the different results 


\section{LICKING CONTRAST}

\section{$R-663$}

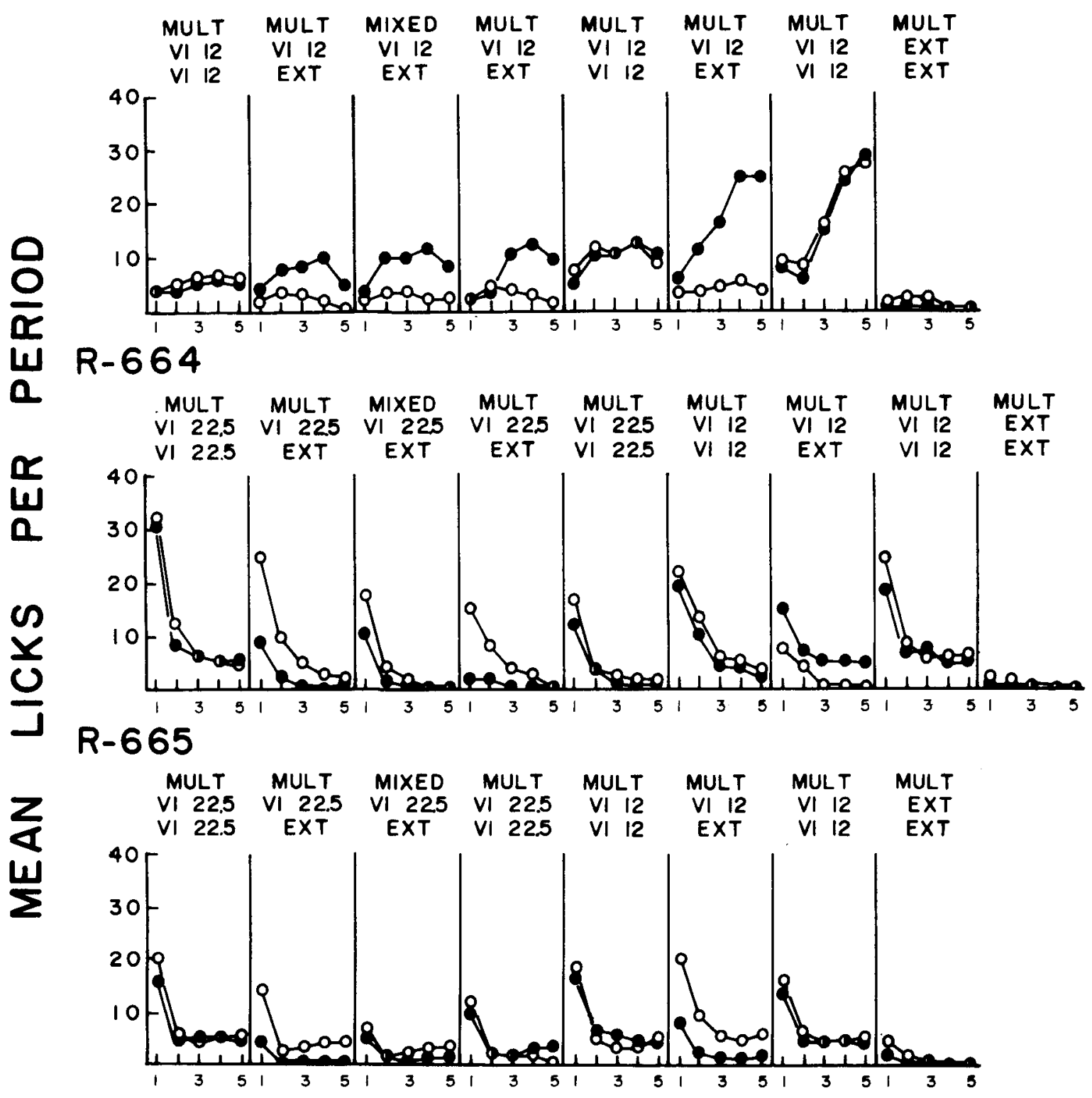

\section{TWELVE-SECOND PERIODS}

Figure 4. Lick distribution during light-plus-tone-on (open circles) and light-plus-tone-off (closed circles) components of the last five sessions of each condition.

of Experiments 1 and 2 is that the increased lick rates in the constant component of the MULT FR 10 EXT schedule in Experiment 1 were simply due to the increased water intake during that component (which in turn was reinforcing the increased lick rates) rather than to the decreased licking (and water intake) in the changed components. This seems unlikely, since lick rates returned to baseline levels when the MULT
FR 10 FR 10 schedule was reinstated. If the increased lick rates had been controlled by the increased water-reinforcement frequency in the FR component, one would expect the elevated lick rates to be maintained upon return to the MULT FR 10 FR 10 schedule. The fact that rat 665 in Experiment 2 did demonstrate polydipsia contrast on a MULT VI 12-sec EXT schedule also argues against this 
explanation. Since the amount of water consumed during the constant VI 12-sec component was not allowed to vary, increased water reinforcement is not necessary for the appearance of schedule-induced polydipsia contrast.

An alternative explanation concerns the possibility that two different response classes were being studied in the two experiments. In Experiment 1, the response class under study was schedule-induced drinking, a response class which must include unrestricted ingestion of water. In Experiment 2, the response class being studied was schedule-induced licking, since water ingestion was restricted. The results of Experiment 2 seem to indicate that the occurrence of positive behavioral contrast with schedule-induced licking depends upon the amount of water available for ingestion during each interval. This argument is supported by the fact that rat 665 demonstrated positive contrast on a VI 12-sec water schedule, but not on a VI 22.5-sec schedule (which provides less available water per interval).

In summary, Experiment 1 demonstrated that positive behavioral contrast occurs with scheduleinduced polydipsia when access to water is relatively unlimited. These findings confirm those of Allen and Porter (1975), and indicate that other instances (Allen et al., 1975; Jacquet, 1972; Porter et al., 1975) of contrast-like effects with schedule-induced polydipsia can probably be attributed to the operations which produce behavioral contrast. The results of Experiment 2 indicate that when the behavior being studied is schedule-induced licking, the appearance of positive behavioral contrast may depend upon the schedule of water availability. The specific conditions under which behavioral contrast will and will not occur with schedule-induced licking remain to be determined.

\section{REFERENCES}

Allen, J. D., \& Porter. J. H. Demonstration of behavioral contrast with adjunctive drinking. Physiology and Behavior. 1975, 15, 511-515.

Allen, J. D., Porter, J. H., \& Arazie, R. Schedule-induced drinking as a function of percentage reinforcement. Journal of the Experimental A nalysis of Behavior, 1975, 23, 223-232.

FALK, J. L. Conditions producing psychogenic polydipsia in animals. Annals of the New York Academy of Sciences, 1969, 157. 569-593.

FALK, J. L. Theoretical review: The nature and determinants of adjunctive behavior. Physiology and Behavior, 1971, 6, 577-587.

Gonzalez, R. C., \& Champlin, G. Positive behavioral contrast, negative simultaneous contrast and their relation to frustration in pigeons. Journal of Comparative and Physiological Psychology. 1974, 87, 173-187.

JaCQUET, Y. F. Schedule-induced licking during multiple schedules. Joumal of the Experimental Anahysis of Behavior, 1972, 17, 413-423.

Mendelson, J., \& Chillag, D. Tongue cooling: A new reward for thirsty rodents. Science, 1970, 170, 1418-1421.

PEAR, J. J., \& WILKIE, D. M. Contrast and induction in rats on multiple schedules. Journal of the Experimental Analysis of Behavior, 1971, 15, 289-296.

Porter, J. H., Arazie. R., Holbrook, J. W., Cheek, M. S., \& Allen, J. D. The effects of variable and fixed second-order schedules on schedule-induced drinking in the rat. Physiology and Behavior, 1975, 14. 143-149.

Premack, P. On some boundary conditions of contrast. In J. Tapp (Ed.), Reinforcement and behavior. New York: Academic Press. 1969. Pp. 120-145.

REYNoLds, G. S. Behavioral contrast. Journal of the Experimental A nalvsis of Behavior, 1961. 4. 57.71.

SEGAL, E. F. Transformation of polydipsia drinking into operant drinking: A paradigm? Psychonomic Science, 1969, 16, 133-135.

Scull, J., DAvies, K., \& AmSEl, A. Behavioral contrast and frustration effect in multiple and mixed fixed-interval schedules in the rat. Journal of Comparative and Physiological Psychology, $1970,71,478-483$.

Terrace, H. S. Discrimination learning, the peak shift, and behavioral contrast. Journal of the Experimental Analysis of Behavior. 1\%8, 11, 727-741.

(Received for publication September 10, 1976; revision accepted February 5, 1977.) 